\title{
Pharmacophore feature-based virtual screening for finding potent GSK-3 inhibitors using molecular docking and dynamics simulations
}

\author{
Navneet Chauhan ${ }^{*}$, Anuradha Gajjar ${ }^{2}$, Syed Hussain Basha ${ }^{3}$ \\ ${ }^{1}$ Department of Pharmaceutical Chemistry, Institute of Pharmacy, Nirma University, Ahmedabad 382 481, Gujarat, India; 2 Department \\ of Pharmaceutical Chemistry, Ramanbhai Patel College of Pharmacy, Charotar University of Science and Technology, Changa 388 421, \\ Gujarat, India; Innovative Informatica Technologies, Hyderabad 500 049, Telangana, India; Navneet Chauhan - E-mail: \\ navneetscientific@gmail.com; Mob: +918511255395; *Corresponding author
}

Received November 9, 2016; Accepted November 21, 2016; Published November 30, 2016

\begin{abstract}
:
Glycogen synthase kinase-3 (GSK-3) is a multitasking serine/threonine protein kinase, which is associated with the pathophysiology of several diseases such as diabetes, cancer, psychiatric and neurodegenerative diseases. Tideglusib is a potent, selective, and irreversible GSK-3 inhibitor that has been investigated in phase II clinical trials for the treatment of progressive supranuclear palsy and Alzheimer's disease. In the present study, we performed pharmacophore feature-based virtual screening for identifying potent targetspecific GSK-3 inhibitors. We found 64 compounds that show better GSK-3 binding potentials compared with those of Tideglusib. We further validated the obtained binding potentials by performing 20-ns molecular dynamics simulations for GSK-3 complexed with Tideglusib and with the best compound found via virtual screening in this study. Several interesting molecular-level interactions were identified, including a covalent interaction with Cys199 residue at the entrance of the GSK-3 active site. These findings are expected to play a crucial role in the binding of target-specific GSK-3 inhibitors.
\end{abstract}

Keywords: Docking, GSK-3, Molecular dynamics, Tideglusib, Virtual screening.

\section{Background:}

Glycogen synthase kinase-3 (GSK-3) is a multitasking serine/threonine protein kinase. Structurally, GSK-3 is a twodomain kinase fold, with a $\beta$-strand domain and an $\alpha$-helix domain. The $\beta$-strand domain (residues $25-138$ ) is located at the $\mathrm{N}$-terminal end and comprises seven antiparallel $\beta$-strands interrupted by a short helix (residues 96-102) comprising highly conserved residues. The Arg96 residue is involved in domain alignment, whereas the Glu97 residue that forms a salt bridge with Lys 85 plays a crucial role in the catalytic activity of the enzyme. The $\alpha$-helical domain comprises residues 139-343 forms at the C-terminal end. The catalytic site is located at the interface of the $\alpha$-helical and $\beta$-strand domains and is surrounded by a hinge region and a glycine-rich loop. The activation loop is formed with residues 200-226 near the substrate binding site [1].

GSK-3 is associated with the pathophysiology of several diseases such as diabetes, cancer, psychiatric and neurodegenerative

ISSN 0973-2063 (online) 0973-8894 (print) diseases. The GSK-3 inhibitors developed so far failed in the preclinical or clinical stages because of limited specificity and unfavorable off-target effects [2]. Tideglusib, a small molecule drug of the thiadiazolidinone (TDZD) class, is the only known GSK-3 inhibitor for which the data obtained in clinical trials support its potential use in the treatment of Progressive Supranuclear Palsy (PSP), and Alzheimer's Disease (AD) [3-5]. However, recently, the Food and Drug Administration (FDA) discontinued approval of this compound because of safety and efficacy issues [6]. Some other independent studies have provided evidence that although this compound is safe, it does not provide clinical benefits, especially to mildly affected patients [7]. In most cases, clinical benefits of Tideglusib are lowered because of its inability to bind tightly and inhibit a specific drug target. In the present study, via computational methods, we performed virtual screening based on pharmacophore features using the Tideglusib structural features for identifying targetspecific GSK-3 inhibitors with better binding potentials. 


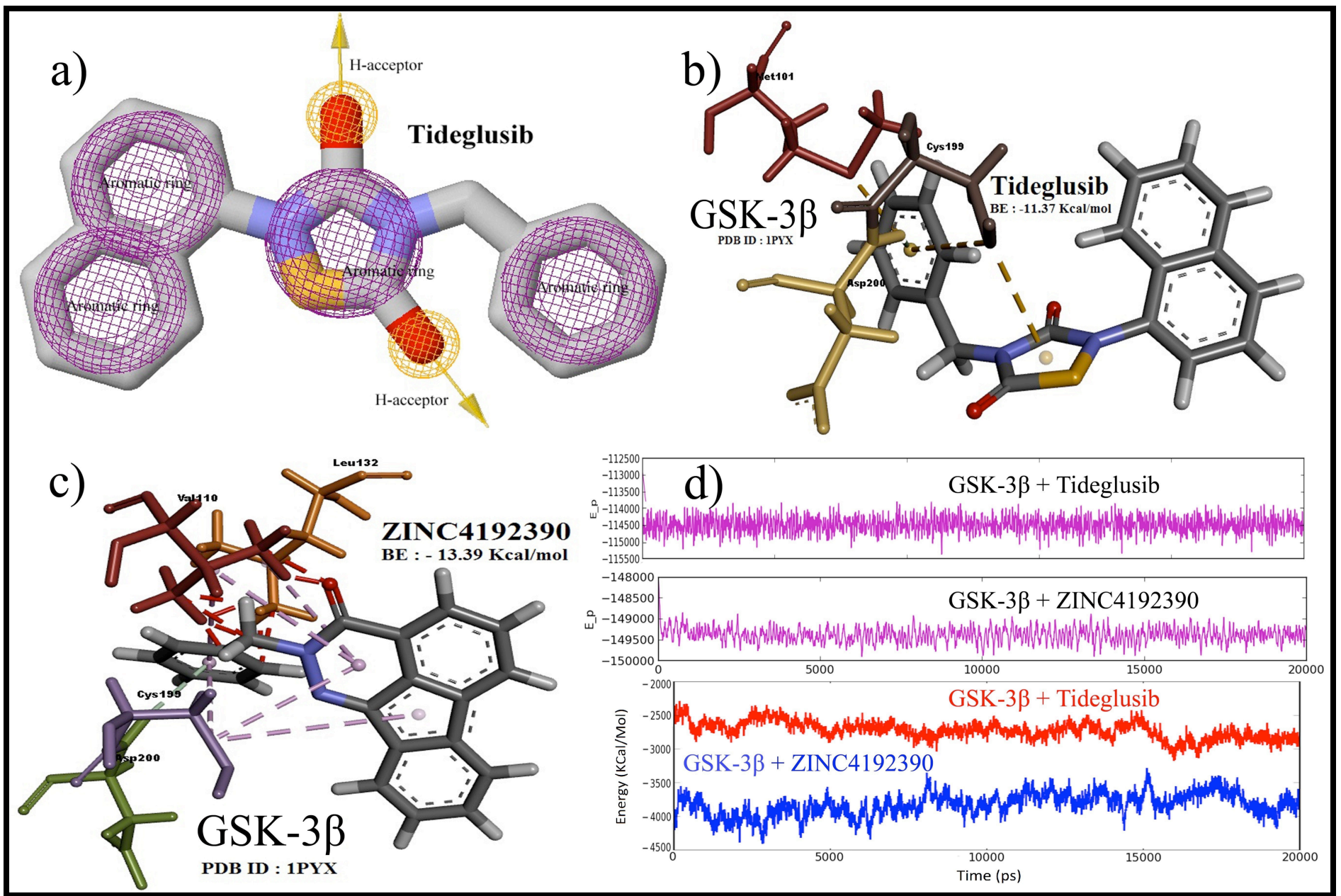

Figure 1 a) Pharmacophore features of Tideglusib, which was used for finding structurally similar compounds for virtual screening; $\mathbf{b}$ ) docking snapshot of Tideglusib at the binding site of GSK-3 with a binding energy (BE) of $-11.37 \mathrm{kcal} / \mathrm{mol}$ showing covalent interaction with Cys199 and hydrogen bonds with Met101 and Asp200 residues; c) docking snapshot of ZINC4192390 compound at the binding site of GSK-3 with a binding energy of $-13.39 \mathrm{kcal} / \mathrm{mol}$ showing covalent interaction with Cys199 and hydrogen bonds with Val110, Leu132, and Asp200 residues; and d) MD simulation energy plots for the GSK-3 complexed with Tideglusib and ZINC4192390.

\section{Methodology:}

Accelrys Discovery studio visualizer v4.0 [8] was used to visualize the receptor, ligand structures, hydrogen-bonding network, bond lengths and to render images. The ZINCPharmer online server [9] was used to identify structurally similar compounds using the pharmacophore features of Tideglusib. ArgusLab v4.0.1[10] was the primary docking program used in this study for virtual screening with semi-flexible docking. A GSK-3 receptor (PDB ID: 1PYX) and ligands were prepared for virtual screening by adding the hydrogen atoms and by removing all water molecules that were co-crystallized with this target protein. The addition of new hydrogen atoms ensures an accurate determination of the ionization states of amino acid residues. The energy of the protein structure was then minimized using the CHARMM (Chemistry at Harvard Macromolecular Mechanics) force field in the Discovery studio package, and the ISSN 0973-2063 (online) 0973-8894 (print) obtained structure was saved in a .pdb file format for further studies. Additionally, ligands were prepared by first removing the hybridization errors in the molecules and then adding the missing hydrogen bonds in order to check the fidelity of all bonds in the compounds. All prepared compounds were saved in the .mol format for further docking studies after a geometry optimization stage with the Universal Force Field (UFF) using a protocol similar to the protocols followed in previous studies [1113]. The energy-scoring grid box was centered at the active ligand binding site location and its dimensions were set to $60 \AA$ along each axis $(x, y$, and $z)$. The grid spacing was $0.40 \AA$, and the default atomic salvation parameters were used. The grid was assigned such that the active site of GSK-3 was enclosed by the three-dimensional grid box. "Ascore" scoring function with all docking parameters set to the default values was selected as the docking engine in ArgusLab. Following the default protocol, the 


\section{Open access}

details of which can be found in previous studies, [15] each molecular dynamics (MD) simulation was conducted for 20-ns using the Desmond module v3.6 [14] from Schrodinger, Inc. For a brief period, the OPLS2005 force field [16] was used to simulate the predefined three-site transferable intermolecular potential (TIP3P) water model [17]. An orthorhombic periodic boundary conditions solvent box buffered at a distance of $10 \AA$ setup to specify the shape and size of the water simulation box. Boundary conditions box volume was calculated to be $478,000 \AA^{3}$. To electrically neutralize the system, appropriate counter ions such as $\mathrm{Cl}^{-}$and $\mathrm{Na}^{+}$were placed randomly in the solvated system.
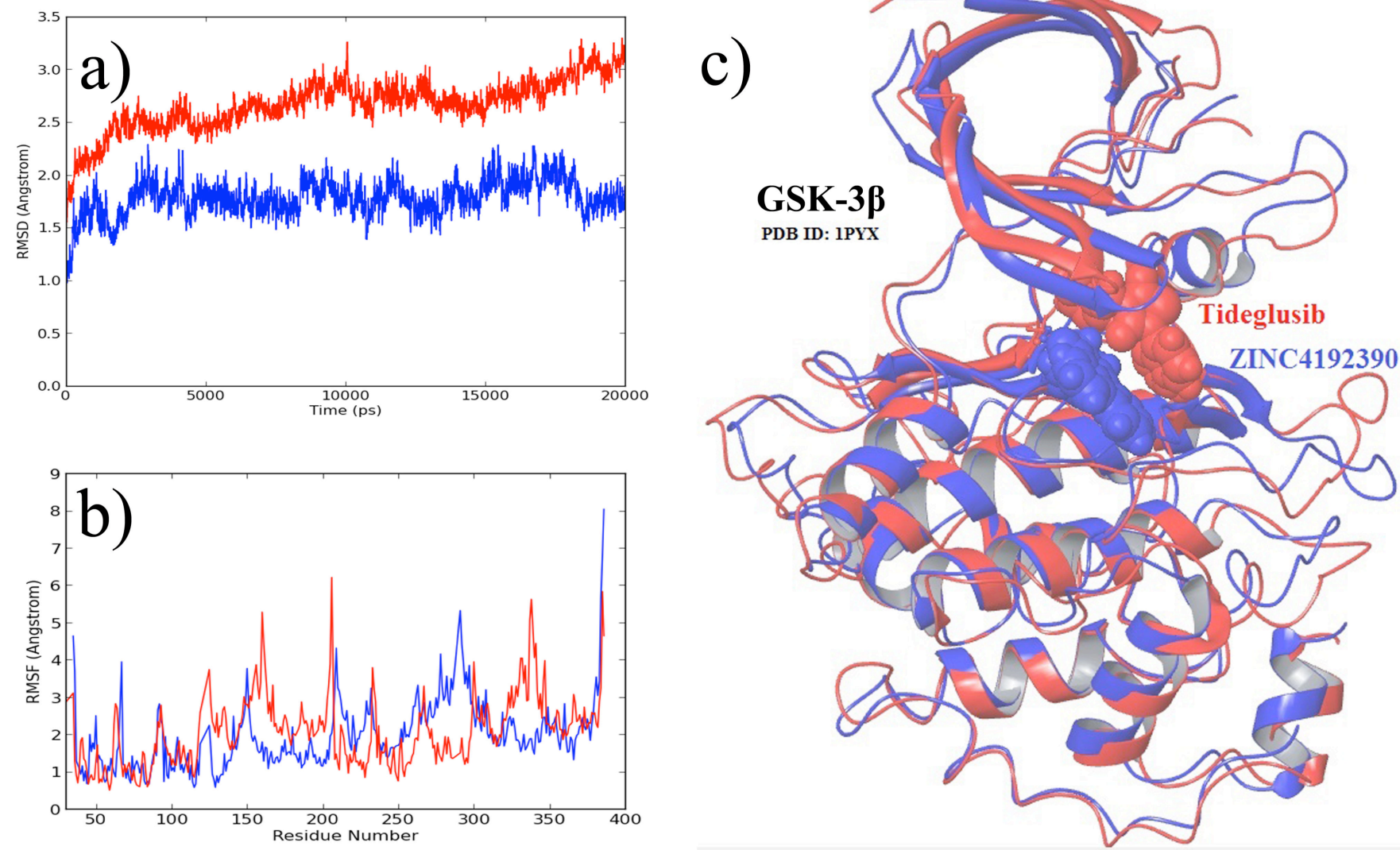

Figure 2 a) Root mean square deviation (RMSD) of GSK-3 protein in presence of Tideglusib (red) and in presence of ZINC4192390 compound (blue) b) Root mean square fluctuations (RMSF) of GSK-3 protein in presence of Tideglusib (red) and in presence of ZINC4192390 compound (blue) c) Superimposition of the final snapshots of MD simulations.

\section{Results and Discussion:}

Pharmacophore search

The structure of the Tideglusib compound was initially downloaded from the ZINC database (ZINC13985228) in the .mol2 format and its energy was then minimized using the CHARMM force field implemented in the Accelrys discovery studio. The obtained relaxed structure was saved in the .pdb format. The saved .pdb file was then uploaded to the ZINCPharmer online server in order to identify the pharmacophore features of the compound. Four aromatic rings with a radius of $1.10 \AA$ were identified to be hydrophobic, and two hydrogen-bond acceptor side chains with a radius of $0.50 \AA$ on the core aromatic ring of the compound were observed as shown in (Figure1a). The identified pharmacophore features of the query molecule were then submitted to the online server for searching structurally similar compounds with matching ISSN 0973-2063 (online) 0973-8894 (print) pharmacophore features. A total of 416 compounds out of 17,818,291 commercially available small molecules exhibited pharmacophore features similar to those of Tideglusib; the details of these 416 compounds were saved as a database of compounds in the .sdf file format for further virtual screening studies.

\section{Virtual Screening}

For virtual screening, a separate database comprising the above mentioned 416 compounds was created. Including Tideglusib, a total of 417 compounds were submitted for virtual screening in the ArgusLab software using the "Dock a database" option. All compounds docked in the active site of GSK-3 with binding energies ranging from -7.71 to $-13.39 \mathrm{kcal} / \mathrm{mol}$. Tideglusib was docked with a binding energy of $-11.37 \mathrm{kcal} / \mathrm{mol}$ by forming a covalent interaction with Cys199 and hydrogen bonds with Met101 and Asp200, as shown in (Figure1b). A total of 63

\section{BIOMEDICAL}


compounds exhibited better binding potentials (more negative binding energy) compared with that of Tideglusib. The ZINC4192390 compound was identified to be the best binding compound, with a binding energy of $-13.39 \mathrm{kcal} / \mathrm{mol}$. This high binding energy resulted from a strong covalent interaction of the Cys199 and hydrogen bonds along with pi-stacking with the Val110, Leu132, and Asp200 residues, as shown in (Figure 1c). The chemical structures of Tideglusib and ZINC4192390 are presented in the Supplementary Information.

Previous studies have reported that the Cys199 residue at the entrance of the GSK-3 active site plays a crucial role in binding Tideglusib to the receptor [18]. This hypothesis has been proved by the studies in which Tideglusib failed to show the inhibition on a panel of kinases that contain a homologous cysteine in the Cys199 location in their active sites, signifying that compounds which are able to tightly bind to this particular residue show a target specific inhibition mechanism [19]. Based on this crucial fact, it is likely that our present proposed compound ZINC4192390 (2-benzylindeno[1,2,3-de]phthalazin-3(2H)-one) has the potential to inhibit specifically target GSK-3 because the binding affinity of GSK-3 is higher than that of Tideglusib.

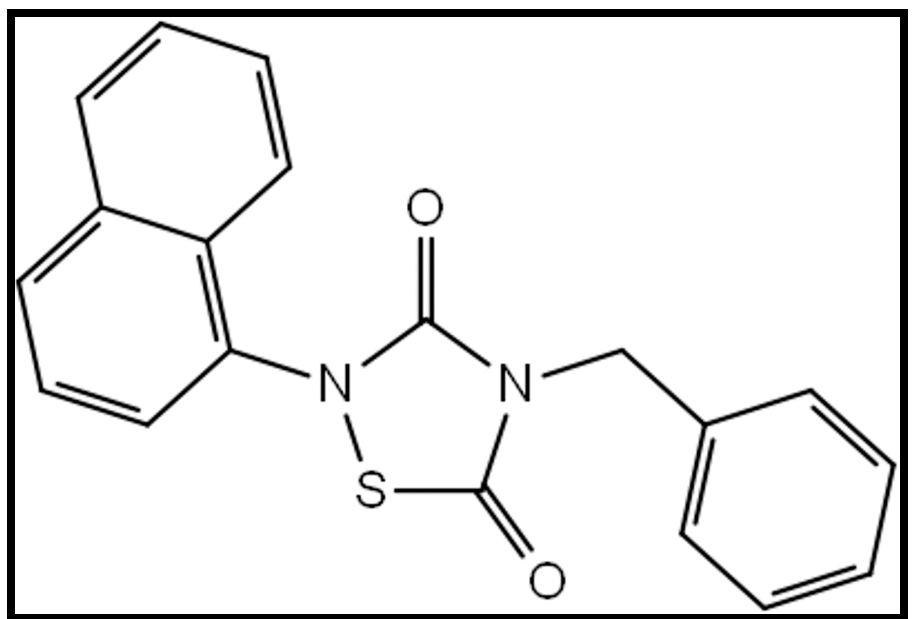

Figure 3: Chemical structure of Tideglusib.

\section{Simulations}

MD simulations were performed to confirm the binding energy and molecular level interactions determined by virtual screening. Initially, we performed individual MD simulations for GSK-3 complexed with Tideglusib and with the best compound identified via virtual screening in this study i.e., ZINC4192390 (2benzylindeno [1,2,3-de]phthalazin-3(2H)-one). Energy plots of GSK-3 complexed with Tideglusib and with the ZINC4192390 compound for 20-ns MD simulations are shown in (Figure 1d). An inspection of the graph clearly shows that our virtual screening predictions are in good agreement with the results for the simulated receptor-ligand complexes.

According to virtual screening, the GSK-3 complexed with Tideglusib showed a binding energy of $-11.37 \mathrm{kcal} / \mathrm{mol}$ whereas ISSN 0973-2063 (online) 0973-8894 (print) the GSK-3 complexed with ZINC4192390 showed a binding energy of $-13.39 \mathrm{kcal} / \mathrm{mol}$; this energy difference shows the same trend shown by the simulated energies of $-114500 \mathrm{kcal} / \mathrm{mol}$ and $149500 \mathrm{kcal} / \mathrm{mol}$, which were observed for the total GSK-3 and Tideglusib system (protein + ligand + solvated water + ions) and for the GSK-3 and ZINC4192390 system, respectively. Therefore, it is clear that the ZINC4192390 compound exhibits a much better binding potential for GSK-3 compared with Tideglusib. Furthermore, this trend was confirmed when we analyzed the simulated energies of the GSK-3 protein in the presence of Tideglusib (red) and thereby obtained a simulated energy of $2500 \mathrm{Kcal} / \mathrm{mol}$. Following a similar procedure for the ZINC4192390 compound (blue), the obtained average simulated energy was $-4000 \mathrm{Kcal} / \mathrm{mol}$.

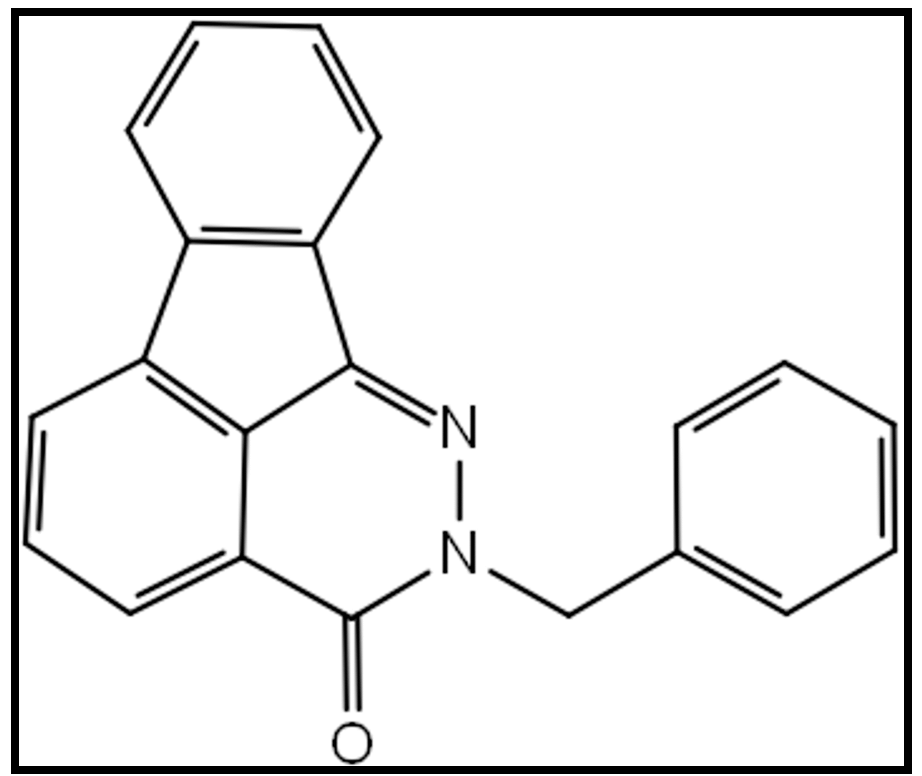

Figure 4: Chemical structure of ZINC4192390 (2-benzylindeno [1,2,3de]phthalazin-3(2H)-one).

To further validate the stability of the GSK-3 complexed with the ZINC4192390 compound compared with that of the GSK-3 complexed with Tideglusib, we analyzed the root mean square deviation (RMSD) (Figure 2a) and the root mean square fluctuation (RMSF) (Figure 2 b) of the GSK-3 protein during 20-ns MD simulations. An inspection of the graphs in (Figure 2) clearly shows that the GSK-3 complexed with ZINC4192390 is much more stable than the GSK-3 complexed with Tideglusib. Moreover, as shown in (Figure 2c), superimposition of the MD simulation snapshots revealed that the ZINC4192390 compound was located much closer to the Cys199 residue, favoring the formation of a stronger binding complex than that obtained with Tideglusib.

\section{Conclusion:}

In this study, we identified ZINC4192390 (2-benzylindeno [1,2,3de]phthalazin-3(2H)-one) as a potential target-specific GSK-3 inhibitor. Several molecular interactions were observed for this 


\section{Open access}

compound, including a strong covalent interaction with the Cys199 residue at the entrance of the GSK-3 active site. This interaction plays a crucial role in the target-specific inhibition mechanism, which may have a potential to improve selectivity of the inhibition over other kinases. The results from this study are expected to provide valuable insights into strategies aimed at optimizing molecules similar to the leading Tideglusib inhibitor compound with better binding potentials and for further evaluation toward the discovery of potential GSK-3 inhibitors.

\section{Conflict of Interest:}

The authors declare that they have no conflict of interest.

Acknowledgements N. Chauhan gratefully acknowledges the Head, Department of Pharmaceutical Chemistry, Saraswati Institute of Pharmaceutical Sciences, Gandhinagar, India, for providing necessary facilities required to execute the doctoral research work.

\section{References:}

[1] Ter Haar E et al. Nat Struct Biol. 2001 8:593 [PMID:11427888].

[2] Eldar-Finkelman H et al. Biochim Biophys Acta 2010 1804:598 [PMID:19770076].

[3] Eldar-Finkelman H \& Martinez A, Front Mol Neurosci. 2011 4:32 [PMID: 22065134].

[4] Serenó L et al. Neurobiol Dis. 2009 35:359 [PMID: 19523516].
[5] Morales-Garcia JA et al. ACS Chem Neurosci. 2012 3:963 [PMID: 23173075].

[6] del Ser T et al. J Alzheimers Dis. 2013 33:205. [PMID: 22936007].

[7] del Ser T, Alzheimers Dement. 2010 6:S147.

[8] Accelrys Software Inc, Discovery studio Visualizer, Version 4.0 http:/ / accelrys.com/

[9] Koes DR et al. PLoS One 2015 10:e0134697 [PMID: 26258606].

[10] Thompson MA: ArgusLab 4.0.1. Seattle, WA: Planaria Software LLC [http:/ / www.arguslab.com].

[11] Basha SH et al. BMC Complement Altern Med. 2013 13:85 [PMID:23587166].

[12] Basha SH \& Prasad RN BMC Res Notes 2012 5:105 [PMID: 22340192].

[13] Rao CM et al. Bioinformation 2015 11:322 [PMID: 26339147].

[14] Desmond Molecular Dynamics System, Version 3.6, D.E. Shaw Research, New York, NY (2013) Maestro-Desmond Interoperability Tools, Version 3.6, Schrödinger, New York, NY, 2013.

[15] Reddy SV et al. J Biomol Struct Dyn. 2015 33:2695 [PMID: 25671592].

[16] Jorgensen WL et al. J Chem Phys. 1983 79:926.

[17] Jorgensen WL et al. J Am Chem Soc. 1996 118:11225.

[18] Martinez A et al. J Med Chem. 2005 48:7103 [PMID:16279768].

[19] Domínguez JM et al. J Biol Chem. 2012 287:893 [PMID:22102280].

Edited by $P$ Kangueane Citation: Chauhan et al. Bioinformation 12(10): 391-395 (2016) License statement: This is an Open Access article which permits unrestricted use, distribution, and reproduction in any medium, provided the original work is properly credited. This is distributed under the terms of the Creative Commons

Attribution License 